\title{
Electron Counting Statistics and Coherent States of Electric Current
}

\author{
Leonid S. Levitov, Hyunwoo Lee \\ Physics Department, Massachusetts Institute of Technology, \\ 12-112, 77 Massachusetts Ave., Cambridge, MA 02139 \\ Gordey B. Lesovik \\ The Institute for Solid State Physics, Chernogolovka, Moscow region, Russia
}

\begin{abstract}
A theory of electron counting statistics in quantum transport is presented. It involves an idealized scheme of current measurement using a spin $1 / 2$ coupled to the current so that it precesses at the rate proportional to the current. Within such an approach, counting charge without breaking the circuit is possible. As an application, we derive the counting statistics in a single channel conductor at finite temperature and bias. For a perfectly transmitting channel the counting distribution is gaussian, both for zero-point fluctuations and at finite temperature. At constant bias and low temperature the distribution is binomial, i.e., it arises from Bernoulli statistics. Another application considered is the noise due to short current pulses that involve few electrons. We find the time-dependence of the driving potential that produces coherent noise-minimizing current pulses, and display analogies of such current states
\end{abstract}


with quantum-mechanical coherent states.

PACS numbers: 72.10.Bg, 73.50.Fq, 73.50.Td

Typeset using REVTEX 


\section{Contents}

\begin{tabular}{lll}
\hline Introduction & 3 \\
\hline
\end{tabular}

\begin{tabular}{|lr}
\hline II $\quad$ Measuring electric current & 6
\end{tabular}

\begin{tabular}{llr}
\hline III $\quad$ Spin $1 / 2$ as a galvanometer & 9
\end{tabular}

IV Single-channel conductor. General formalism 13

\begin{tabular}{|lr}
\hline $\mathrm{V} \quad$ Equilibrium fluctuations & 16
\end{tabular}

\begin{tabular}{|lll}
\hline VI $\quad$ Statistics of a dc current: quantum shot noise & 18
\end{tabular}

VII Noise due to a voltage pulse: Orthogonality catastrophe 19

\begin{tabular}{|lr}
\hline VIII Coherent states of current & 26 \\
\hline
\end{tabular}

IX Conclusions

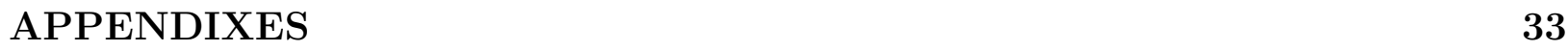

\begin{tabular}{|lll}
\hline A Larmor clock measurement of tunneling time & 34
\end{tabular}

B Bosonization calculation of counting statistics $\quad 38$

\section{INTRODUCTION}

Quantum transport in nanostructures has been a subject of many recent studies [1]. Transport properties like Ohmic conductivity can be understood in terms of the quantum scattering problem in the conductor, which provides a theory of quantum coherence of 
transport [2]. Fluctuations of electric current due to the discreteness of electric charge are intrinsic to quantum transport [3 5 ]. It has been found that current fluctuations have interesting properties reflecting profound aspects of underlying quantum dynamics [6 9]. For example, the quantum noise caused by a dc current is reduced below classical shot noise level [3 9]. This suppression has been understood as an effect of enhanced regularity of transmission events due to Fermi statistics [10]. Besides theoretical interest, such phenomena may lead to applications. Given the development of nano-technologies, the transmission of signals by single- or few-electron pulses will become common, and then one will see the quantum statistics of current working.

In this paper we update the theory of quantum measurement of electric current [11]. Our goal is a complete description of charge fluctuations, rather than developing measurement theory (see Secs. II, III). We shall derive a microscopic formula for electron counting distribution (see Sec. III, Eq. (11), and Sec. IV, Eq. (26)) that can be used for any system, e.g., with an interaction or with a time-dependent potential [12]. As an application, we test the method on the statistics in a single channel ideal conductor for non-equilibrium and equilibrium noise at finite temperature, and for zero-point equilibrium fluctuations (Secs. IV, V). In particular, the fluctuations of a dc current at zero temperature are found to be binomial (Sec. VI) with the probabilities of outcomes related with transmission coefficients of elastic scattering in the system, and with the number of attempts $N=e V t / h$, where $V$ is applied voltage, and $t$ is the time of measurement.

Another property of quantum noise that does not have classical analog is its phase sensitivity [13, 14]. For the current correlator $\left\langle\left\langle j\left(t_{1}\right) j\left(t_{2}\right)\right\rangle\right\rangle_{+}$it results in a periodic sinusoidal dependence on Faraday's flux due to applied voltage, $\Phi=c \int_{t_{1}}^{t_{2}} V(t) d t$, with the period 
$\Phi_{0}=h c / e$. The phase sensitivity manifests in singularities of the low frequency noise power in a junction driven by ac and dc signals together [15].

Even more remarkable is the behavior of current fluctuations due to a pulse of voltage [13,16]. Total charge that flows through the conductor due to a voltage pulse fluctuates in such a way that the mean square fluctuation diverges whenever the flux of the pulse is not an integer: $\varphi=\frac{e}{\hbar} \int_{-\infty}^{\infty} V(t) d t \neq 2 \pi n$. On the other hand, for $\varphi=2 \pi n$ the fluctuation of the transmitted charge is finite (Sec. VII). This result has simple interpretation in terms of the Anderson orthogonality catastrophe theory, since the flux $\varphi$ enters the time dependent scattering matrix of the conductor through the forward scattering amplitude.

With this, one is led to address the issue of current states that minimize the current fluctuations at fixed mean transmitted charge [17,[16]. It is found in Sec. VIII that such states are produced by time-dependent voltage of the form

$$
V(t)= \pm \frac{h}{\pi e} \sum_{k=1}^{n} \frac{\tau_{k}}{\left(t-t_{k}\right)^{2}+\tau_{k}^{2}}, \tau_{k}>0,
$$

a sum of Lorentzian pulses of unit flux each. It is remarkable that the minimal noise due to such sequence of pulses is independent of the pulse positions $t_{k}$ and widths $\tau_{k}$, which leads to obvious parallels with solitons in the theory of non-linear integrable systems. The noise minimizing current states can be compared to the coherent states that minimize the quantum-mechanical uncertainty. Apart from obvious similarity, there is a difference: the coherent current states are many-body time-dependent scattering states. Their role in transport is an interesting subject of future work: one expects that representing many-body states as a superposition of these coherent states has an advantage similar to that provided by coherent states of one particle. 


\section{MEASURING ELECTRIC CURRENT}

Instantaneous measurement is described in quantum mechanics by wavepacket reduction that involves projecting on eigenstates of an observable, represented by a hermitian operator. A different kind of measurement, extended in the time domain, is realized in detectors and other counting devices. It is known that in such cases a certain revision of the measurement description is necessary. A famous example is the theory of photon detectors [18 in quantum optics. Due to Bose statistics, photons entering a photo-counter are correlated in time, and this makes the theory of photon detection a problem of many-particle statistics. For a single normal mode of radiation field the probability $P_{m}$ to count $m$ photons over time $t$ is given by

$$
P_{m}=\frac{(\eta t)^{m}}{m !}\left\langle:\left(a^{+} a\right)^{m} e^{-\eta t a^{+} a}:\right\rangle
$$

where $a^{+}$and $a$ are Bose operators of the mode, $\eta$ is the counter efficiency parameter, and $\langle\ldots\rangle$ stands for the average over a quantum state. The normal ordering : . . : is an important element of the formalism. Physically, it means that, after having been detected, each photon is destroyed, e.g., it is absorbed in the detector. Instead of the probabilities (2), it is more convenient to deal with the generating function

$$
\chi(\lambda)=\sum_{m} P_{m} e^{i \lambda m}
$$

For the single normal mode Eq.(2) leads to

$$
\chi(\lambda)=\left\langle: \exp \eta t\left(e^{i \lambda}-1\right) a^{+} a:\right\rangle .
$$

Eqs.(22,3, (1) account very well for numerous experimental situations [19]. Particularly interesting is the case of a coherent state $|\mathrm{z}\rangle, a|\mathrm{z}\rangle=z|\mathrm{z}\rangle$, where $z$ is a complex number. It 
corresponds to the radiation field of an ideal laser, and with Eq.(蛋) one easily gets Poisson counting distribution,

$$
P_{m}=\frac{(J t)^{m}}{m !} e^{-J t}, \quad J=\eta|z|^{2}
$$

which describes the so-called minimally bunched light source.

Similar to the photon detection, electric measurement is performed on a system containing an enormous number of particles — in this case fermions - and thus one expects the effects of Fermi statistics to be important. Also, the duration of electric measurement is typically much longer than the time it takes the system to transmit one electron by microscopic tunneling, scattering, or diffusion. Apart from these similarities, there is, however, a crucial difference from the photon counting: the number of electrons is not changed by the current measurement, since electric charge is conserved. This has to be contrasted with absorption of photons in photo-counters. Related to this, there is another important difference: at every detection of a photon, its energy $\hbar \omega$ is taken from the radiation field, which makes plain photodetectors insensitive to zero-point fluctuations of electromagnetic field. On the contrary, the measurement of current fluctuation is usually performed without changing energy of the system, which makes the zero-point noise an unavoidable component of any electric measurement [20]. (Let us emphasize that the difference has nothing to do with the type of quantum statistics, Fermi or Bose. Rather it is the difference between the two kinds of measurement, e.g., see [21], where counting of fermions was discussed using an optical-like counter that has to capture an electron in order to detect it.)

In the classical picture, the measurement gives the charge $Q(t)=\int_{0}^{t} j\left(t^{\prime}\right) d t^{\prime}$ transmitted during the measurement time $t$. The probabilities $P_{m}$ of counting $m$ electrons can then be 
obtained by averaging $\delta(Q(t)-m e)$ over the state of the system. In a quantum problem electric current is an operator, and since currents at different moments do not commute, the operator of transmitted charge $\widehat{Q}(t)=\int_{0}^{t} \widehat{j}\left(t^{\prime}\right) d t^{\prime}$ generally does not make any sense. Instead, since we are interested in higher-order statistics of current fluctuations, beyond $\langle\widehat{j}(t)\rangle$ and $\left\langle\left\langle j\left(t_{1}\right) j\left(t_{2}\right)\right\rangle\right\rangle_{+}$, in order to compute electron counting ditribution, we have to include the measuring system in the quantum Hamiltonian. Our approach is motivated by the example of the quantum mechanical systems with strong coupling to macroscopic environment, introduced by Leggett, that can be treated consistently only by adding the "measuring environment" to the quantum problem [22].

For that we introduce a model quantum galvanometer, a spin $1 / 2$ that precesses in the magnetic field $B$ of the current. For a classical system, the rate of precession is proportional to $B(t)$, and $B(t)$ is proportional to the current $I(t): B(t)=$ const $I(t)$. Therefore, the precession angle of the spin directly measures transmitted charge $\delta Q=\int_{0}^{t} I\left(t^{\prime}\right) d t^{\prime}$. We adopt the same measurement procedure for the quantum circuit, i.e., we include in the electron Hamiltonian the vector potential due to the spin:

$$
\vec{A}(r)=-\mu \overrightarrow{\widehat{\sigma}} \times \vec{\nabla} \frac{1}{|r|}
$$

where $\overrightarrow{\hat{\sigma}}=\left(\sigma_{x}, \sigma_{y}, \sigma_{z}\right)$ are Pauli matrices. Thus we obtain a Hamiltonian describing motion of electrons, the measuring spin, and their coupling. Now, according to what has been said, we have to solve dynamics of the spin in the presence of the fluctuating current, find the distribution of precession angles, and then interpret it as a distribution of transmitted charge. Of course, a question remains about the back effect of the spin on the system, as in any other problem of quantum measurement. However, as we find below in (18) and (19), 
only the phase of an electron state is affected by the presence of the spin, not the amplitude. Moreover, the phase will change only for the transmitted, but not for the reflected wave. As a result, the probabilities we obtain do not depend on the coupling constant of the spin. This justifies the assumption that the spin measures charge transfer in a non-invasive way.

It is worth remarking that our scheme resembles the "Larmor clock" approach [23] to the problem of traversal time for motion through a classically forbidden region. In this problem one is interested, e.g., in the time spent by a particle tunneling through a barrier. The Larmor clock approach involves an auxiliary constant magnetic field $B$ added in the classically forbidden region, and a spin $1 / 2$ carried by the particle that interacts with the field: $\mathcal{H}_{\text {int }}=-\widehat{\sigma}_{z} B$. The precesson angle of the spin measures traversal time. Comparing the two approaches is very tutorial: see Appendix A, where the Larmor clock is reviewed.

\section{SPIN 1/2 AS A GALVANOMETER}

Having clarified our motivation, we proceed semi-phenomenologically and choose a new vector potential in the spin-current interaction $-\frac{1}{c} \vec{j} \vec{A}$. We replace the Ampère's long-range form (6) by a model vector potential

$$
\widehat{A}_{i}(r)=\frac{\lambda \Phi_{0}}{4 \pi} \widehat{\sigma}_{z} \nabla_{i} \theta\left(f(r)-f_{0}\right)
$$

concentrated on some surface $S$ defined by the equation $f(r)=f_{0}$. Here $\Phi_{0}=h c / e, \lambda$ is a coupling constant, $f(r)$ is an arbitrary function, and, as usual, the step-function $\theta(x)=1$ for $x>0,0$ for $x<0$. The surface $S$ defines a section of the conductor on which the interaction is localized:

$$
\mathcal{H}_{\text {int }}=\int-\frac{1}{c} \overrightarrow{\hat{j}} \overrightarrow{\widehat{A}} d^{3} r=-\frac{\lambda \hbar}{2 e} \widehat{\sigma}_{z} \widehat{I}_{S},
$$


where $\widehat{I}_{S}=\int_{S} \overrightarrow{\hat{j}} \vec{d} s$, i.e., the spin now is coupled to the total current through the section $S$. With the choice (7) of the vector potential one can study current fluctuations in an arbitrary section of the conductor. Another advantage of the phenomenological Eq.(17) is that it involves only one Pauli matrix, which makes the spin dynamics essentially trivial. The choice of the quantization axis of the spin is arbitrary since (8) will be the only spindependent part of the Hamiltonian. Finally, another advantage of the form (8) is that by switching from the smooth function (6) to the singular form (77) we enforce integer values of counted charge. To understand this, let us note that in the "fuzzy" case (6) the measurement can start at the moment when one of the electrons is located somewhere in the middle of the volume where $A \neq 0$, and then a fractional part of electron charge will be counted. On the contrary, in the "sharp" case (7), the spin responds to the presence of an electron only when it crosses the section $S$. We shall see below in a microscopic calculation that integer values of charge follow automatically from gauge invariance, since the form (17) is a gradient of a scalar.

Thus we come to the Hamiltonian

$$
\widehat{\mathcal{H}}_{\sigma}=\widehat{\mathcal{H}}(\tilde{p}, r), \quad \tilde{p}_{i}=p_{i}-\frac{e}{c} \widehat{A}_{i}
$$

where the spin-dependent $\overrightarrow{\widehat{A}}$ is taken in the form (7). An essential feature of our approach is that we treat the constant $\lambda$ of coupling between the spin and the current as a variable, i.e., we consider the spin precession as a function of the parameter $\lambda$. The reason is that, unlike the photon counting problem, our measurement scheme directly generates the function $\chi(\lambda)$, and then the counting probabilities $P_{m}$ are obtained by reading Eq.(3) backwards.

At this point we are able to formulate our main result. Let us define a new Hamiltonian 


$$
\widehat{\mathcal{H}}_{\lambda}=\widehat{\mathcal{H}}(\tilde{p}, r), \quad \tilde{p}_{i}=p_{i}-\frac{1}{2} \lambda \hbar \nabla_{i} \theta\left(f(r)-f_{0}\right)
$$

simply by supressing $\widehat{\sigma}_{z}$ in Eq.(17). The Hamiltonian $\widehat{\mathcal{H}}_{\lambda}$ involves only quantities of the electron subsystem. Below we show that by measuring precession of the spin coupled to the current, one obtains the quantity

$$
\chi(\lambda)=\left\langle e^{i \widehat{\mathcal{H}}_{-\lambda} t} e^{-i \widehat{\mathcal{H}}_{\lambda} t}\right\rangle
$$

Here the brackets $\langle\ldots\rangle$ stand for averaging over initial state of electrons. Note that $\chi(\lambda)$ is written in terms of a purely electron problem, not involving spin variables. We shall find that the function $\chi(\lambda)$ defines the result of any measurement of the spin polarization after the time $t$ when the spin-current coupling is turned off. Moreover, we shall see that the function (11) has the meaning of a generating function of electron counting distribution, i.e., the Fourier transform of $\chi(\lambda)$ gives counting probabilities, entirely analogous to (3).

Our goal now will be to express evolution of the spin in terms of quantities corresponding to the electron system. The interaction is given by Eqs.(7),(19). Suppose that the measurement started at the moment 0 and stopped at the time $t$, i.e., the spin-current interaction is turned on during the time interval $0<\tau<t$. Let us evaluate the density matrix $\hat{\rho}_{s}(t)$ of the spin, right after it is disconnected from the circuit. We have

$$
\widehat{\rho}_{s}(t)=\operatorname{tr}_{e}\left(e^{-i \widehat{\mathcal{H}}_{\sigma} t} \widehat{\rho} e^{i \widehat{\mathcal{H}}_{\sigma} t}\right)
$$

where $\hat{\rho}$ is initial density matrix $\hat{\rho}_{e} \otimes \widehat{\rho}_{s}$ at $t=0, \widehat{\rho}_{e}$ is initial density matrix of electrons, and $\operatorname{tr}_{e}(\ldots)$ means partial trace taken over electron states, the spin indices left free. In terms of the spin variables, the operator $e^{-i \widehat{\mathcal{H}}_{\sigma} t}$ is a function only of $\widehat{\sigma}_{z}$, and hence it is diagonal in spin: $\left\langle\uparrow\left|e^{-i \widehat{\mathcal{H}}_{\sigma} t}\right| \downarrow\right\rangle=\left\langle\downarrow\left|e^{-i \widehat{\mathcal{H}}_{\sigma} t}\right| \uparrow\right\rangle=0$. In other words, if initially the spin is in a pure state, up or down, it will not precess. For $\hat{\rho}_{s}(t)$ this remark yields: 


$$
\widehat{\rho}_{s}(t)=\left[\begin{array}{cc}
\hat{\rho}_{\uparrow \uparrow}(0) & \chi(\lambda) \widehat{\rho}_{\uparrow \downarrow}(0) \\
\chi(-\lambda) \widehat{\rho}_{\downarrow \uparrow}(0) & \widehat{\rho}_{\downarrow \downarrow}(0)
\end{array}\right] .
$$

Here $\chi(\lambda)=\operatorname{tr}_{e}\left(e^{-i \widehat{\mathcal{H}}_{\lambda} t} \widehat{\rho}_{e} e^{i \widehat{\mathcal{H}}_{-\lambda} t}\right)$, where $e^{-i \widehat{\mathcal{H}}_{\lambda} t}$ is the evolution operator for the problem (10). Now, after the spin degrees of freedom are taken care of by (13), we are left with a purely electron problem, that involves only electron degrees of freedom, but not the spin. By using the cyclic property of the $\operatorname{trace} \operatorname{tr}_{e}(\ldots)$, one can show that $\chi(\lambda)$ in Eq.(13) is identical to (11).

In principle, any entry of a density matrix can be measured, and therefore the quantity $\chi(\lambda)$ is also measurable. In order to make clear the relation of $\chi(\lambda)$ with the distribution of precession angles, let us recall the transformation rule for the spin $1 / 2$ density matrix under rotation by an angle $\theta$ around the $z$-axis:

$$
\mathcal{R}_{\theta}(\widehat{\rho})=\left[\begin{array}{cc}
\hat{\rho}_{\uparrow \uparrow} & e^{-i \theta} \widehat{\rho}_{\uparrow \downarrow} \\
e^{i \theta} \widehat{\rho}_{\downarrow \uparrow} & \widehat{\rho}_{\downarrow \downarrow}
\end{array}\right] .
$$

By combining this with Eq.(3) we write $\widehat{\rho}_{s}(t)$ as

$$
\widehat{\rho}_{s}(t)=\sum_{m} P_{m} \mathcal{R}_{\theta=m \lambda}(\widehat{\rho})
$$

which assigns to $P_{m}$ the meaning of the probability to observe precession angle $m \lambda$. Let us finally note that such interpretation of $P_{m}$ is consistent with what one expects on classical grounds, because for a classical magnetic moment $\vec{\sigma}$ interacting with the current according to (घ), the angle $\theta=\lambda$ corresponds to the precession due to a current pulse carrying the charge of one electron. 


\section{SINGLE-CHANNEL CONDUCTOR. GENERAL FORMALISM}

In order to see Eq.(11) working, let us consider an ideal single channel conductor, i.e., the Schrödinger equation

$$
i \frac{\partial \psi}{\partial t}=\left[\frac{1}{2}\left(-i \frac{\partial}{\partial x}-\frac{\lambda}{2} \delta(x)\right)^{2}+U(x)\right] \psi
$$

in one dimension, where the potential $U(x)$ represents scattering region and the vector potential is inserted according to (77) at the $x=0$ section. In order to describe transport, we shall use scattering states, left and right. Their energy distributions $n_{L(R)}(E)$ are equilibrium Fermi functions with temperature $T$ and chemical potentials shifted by $e V, \mu_{L}-\mu_{R}=e V$, representing a dc voltage.

For the problem (16) one can write time dependent scattering states as

$$
\begin{gathered}
\psi_{L, k}(x, t)=e^{-i E_{k} t} \begin{cases}e^{i k x}+B_{L} e^{-i k x}, & x<-a / 2 \\
e^{i \lambda / 2} A_{L} e^{i k x}, & x>a / 2\end{cases} \\
\psi_{R, k}(x, t)=e^{-i E_{k} t} \begin{cases}e^{-i \lambda / 2} A_{R} e^{-i k x}, & x<-a / 2 \\
e^{-i k x}+B_{R} e^{i k x}, & x>a / 2\end{cases}
\end{gathered}
$$

where $a$ is the width of the barrier, and $A_{L, R}$ and $B_{L, R}$ are the transmission and reflection amplitudes in the absence of the spin vector potential. To make expressions less heavy, we supress electron spin. The phase factors $e^{ \pm i \lambda / 2}$ in (17) are found immediately by observing that the vector potential in the Schrödinger equation can be eliminated by the gauge transformation $\psi(x) \rightarrow \exp (i \lambda / 2 \theta(x)) \psi(x)$. The scattering amplitudes form a unitary matrix:

$$
\widehat{S}_{\lambda}=\left[\begin{array}{cc}
e^{i \lambda / 2} A_{L} & B_{R} \\
B_{L} & e^{-i \lambda / 2} A_{R}
\end{array}\right]
$$

We will study the range of small $T, e V \ll E_{F}$, when only the states close to the Fermi level are important. In this case, there is an additional simplification because the states 
near Fermi energy have almost linear dispersion, and thus all wavepackets travel with the speed $v_{F}$ without changing shape. Then, following Landauer and Martin [8], instead of the usual scattering states (17), it is convenient to use their Fourier transform. By ignoring the energy-dependence of $A_{L, R}$ and $B_{L, R}$, which is equivalent to saying that the scattering time is negligible, and assuming that the dispersion is stricktly linear, one obtains the representation of scattering in terms of time-dependent scattering wave packets

$$
\begin{gathered}
\psi_{L, \tau}(x, t)= \begin{cases}\delta\left(x_{-}\right), & t<\tau \\
e^{i \lambda / 2} A_{L} \delta\left(x_{-}\right)+B_{L} \delta\left(x_{+}\right), & t>\tau\end{cases} \\
\psi_{R, \tau}(x, t)= \begin{cases}\delta\left(x_{+}\right), & t<\tau \\
e^{-i \lambda / 2} A_{R} \delta\left(x_{+}\right)+B_{R} \delta\left(x_{-}\right), & t>\tau\end{cases}
\end{gathered}
$$

where $x_{ \pm}=x \pm v_{F}(t-\tau)$. Here $\tau$ is the packet arrival moment, at which it is scattered. It is straightforward to verify orthogonality of the states (19). Any time-dependent electron state can be written as a superposition of the states (19), with the arrival time $\tau$ serving in this representation as a label in the continuum of states, like $k$ in (17). The assumption that the scattering amplitudes are energy-independent (and thus the scattering takes no time) is equivalent to replacing the barrier $U(x)$ of finite width by $U_{0} \delta(x)$ and is consistent with the closeness of relevant energies to $E_{F}$.

Second-quantized, electron states (19) lead to $\widehat{\psi}(x, t)=\widehat{\psi}_{L}(x, t)+\widehat{\psi}_{R}(x, t)$ with

$$
\widehat{\psi}_{L(R)}(x, t)=\sum_{\tau} \psi_{L(R), \tau}(x, t) \widehat{c}_{1(2), \tau}
$$

where $c_{1, \tau}$ and $c_{2, \tau}$ are canonical Fermi operators corresponding to the states (19), the left and the right respectively. One checks that fermionic commutation relations for $c_{1(2), \tau}$,

$$
c_{i, \tau}^{+} c_{j, \tau^{\prime}}+c_{j, \tau^{\prime}} c_{i, \tau}^{+}=\delta_{i j} \delta\left(\tau-\tau^{\prime}\right)
$$




$$
c_{i, \tau} c_{j, \tau^{\prime}}+c_{j, \tau^{\prime}} c_{i, \tau}=0 \quad, c_{i, \tau}^{+} c_{j, \tau^{\prime}}^{+}+c_{j, \tau^{\prime}}^{+} c_{i, \tau}^{+}=0
$$

yield the usual commutation relations for $\psi_{L(R)}(x, t)$. From that one finds the meaning of the summation in 20 ): $\sum_{\tau} \ldots=\int_{-\infty}^{\infty} \ldots d \tau$. Mathematically, in this paragraph we defined second-quantized $\psi(x)$ in (16).

The advantage of introducing the basis of the wavepackets $(19),(20)$ is that now it is straightforward to write the many-particle evolution operator through the single-particle scattering matrix $\widehat{S}_{\lambda}$ :

$$
e^{-i \widehat{\mathcal{H}}_{\lambda} t}=\exp \int_{0}^{t} d \tau \sum_{i j} \ln \left[\widehat{S}_{\lambda}\right]_{i j} c_{i, \tau}^{+} c_{j, \tau}
$$

where $\widehat{S}_{\lambda}$ is given by (18). To verify $(23)$, let us note that in the wavepacket representation (19), according to Eqs. (21), Fermi correlations occur only for the pairs of left and right states that scatter at the same instant of time. For each of such pairs the evolution operator $e^{-i \widehat{\mathcal{H}}_{\lambda} t}$ is $\widehat{1}$ if both states are occupied or both are empty, otherwise it is given by the single-particle scattering matrix (18).

Using similar arguments, we compute

$$
e^{i \widehat{\mathcal{H}}_{-\lambda} t} e^{-i \widehat{\mathcal{H}}_{\lambda} t}=\exp \int_{0}^{t} d \tau \sum_{i j} \widehat{W}_{i j} c_{i, \tau}^{+} c_{j, \tau}
$$

where $e^{\widehat{W}}=\widehat{S}_{-\lambda}^{-1} \widehat{S}_{\lambda}$ is readily obtained from (18):

$$
e^{\widehat{W}}=\left[\begin{array}{cc}
e^{i \lambda}\left|A_{L}\right|^{2}+\left|B_{L}\right|^{2} & 2 i \sin \lambda \bar{A}_{L} B_{R} \\
2 i \sin \lambda \bar{B}_{R} A_{L} & e^{-i \lambda}\left|A_{R}\right|^{2}+\left|B_{R}\right|^{2}
\end{array}\right]
$$

Using unitarity of $e^{\widehat{W}}$ and commutation rules for $c_{\alpha, \tau}$ one can rewrite (24) in terms of normal ordering:

$$
e^{i \widehat{\mathcal{H}}_{-\lambda} t} e^{-i \widehat{\mathcal{H}}_{\lambda} t}=: \exp \int_{0}^{t} d \tau \sum_{i j}\left[e^{\widehat{W}}-1\right]_{i j} c_{i, \tau}^{+} c_{j, \tau}
$$


This form is ready to be plugged into Eq.(11) and averaged over the initial state. Let us note the striking similarity of two formulas obtained by different means: the fermionic Eq.(26) and the bosonic Eq.(4).

Also, let us mention that the periodicity of the matrix (25) in $\lambda$ ensures periodicity of $\chi(\lambda)$, and thus guarantees integer values of charge.

\section{EQUILIBRIUM FLUCTUATIONS}

Let us start with a simple one-particle example. Consider a particle in the state $c_{1, \tau}^{+}|\mathrm{vac}\rangle$, which corresponds to scattering at the moment $\tau$. In this case, from (26) and (11) one gets

$$
\chi(\lambda)= \begin{cases}e^{i \lambda}|A|^{2}+|B|^{2} & \text { for } 0<\tau<t \\ 1 & \text { otherwise }\end{cases}
$$

$|A|=\left|A_{L}\right|=\left|A_{R}\right|,|B|=\left|B_{L}\right|=\left|B_{R}\right|$. Evidently, according to Eq.(3), this simply means that for the scattering occurring during operation of the detector, the counting probabilities are identical to the one-particle scattering probabilities, as it should be expected.

Now, we consider current fluctuations in an equilibrium Fermi gas. First, let us assume perfect transmission: $B_{L(R)}=0$. Then Eq.(25) gives $\widehat{W}=i \lambda \sigma_{z}$, and thus Eq.(24) becomes

$$
e^{i \widehat{\mathcal{H}}_{-\lambda} t} e^{-i \widehat{\mathcal{H}}_{\lambda} t}=\exp i \lambda \int_{0}^{t}\left(c_{1, \tau}^{+} c_{1, \tau}-c_{2, \tau}^{+} c_{2, \tau}\right) d \tau
$$

i.e., the right and the left states separate.

We observe that averaging of (28) over the Fermi ground state is identical to that performed in the orthogonality catastrophe calculation [24,25]. Hence, averaging of (28) can be done by using the bosonization method [26] that replaces the fermionic Hamiltonian by a bosonic one. (The calculation is described in Appendix B.) In the bosonized representation one has to do a simple gaussian average, which gives 


$$
\chi(\lambda)=e^{-\tilde{\lambda}^{2} f(t, T)}
$$

where $\tilde{\lambda} / 2 \pi+1 / 2=\lfloor\lambda / 2 \pi+1 / 2\rfloor$, with $\lfloor\ldots\rfloor$ being the fractional part. The function

$$
\begin{aligned}
f(t, T) & =\left\langle\left\langle\left(\int_{0}^{t} c_{1, \tau}^{+} c_{1, \tau} d \tau\right)^{2}\right\rangle\right\rangle=-\operatorname{Re} \frac{1}{4} \int_{0}^{t} \int_{0}^{t} \frac{T^{2} d t_{1} d t_{2}}{\sinh ^{2}\left(\pi T\left(t_{1}-t_{2}-i \delta\right)\right)} \\
& =\frac{1}{2 \pi^{2}} \ln \left(\frac{1}{\pi T \delta} \sinh \pi T t\right)=\left\{\begin{array}{l}
\frac{1}{2 \pi^{2}} \ln t / \delta \quad, \delta \ll t \ll \hbar / T \\
T t / h-\frac{1}{2 \pi^{2}} \ln 2 \pi T \delta, \quad t \gg \hbar / T
\end{array}\right.
\end{aligned}
$$

where $\delta$ is an ultraviolet cutoff time, of the order of $\hbar / E_{F}$. At long times, according to (3), this leads to gaussian counting statistics.

As a side remark, the distribution given by Eq.(29) also gives a solution to another problem: the statistics of the number of fermions inside a segment of fixed length in one dimension. The relation is immediately obvious after one assigns to $\tau$ in Eq.(28) the meaning of a coordinate on a line. Thus, in this problem the statistics are gaussian as well.

Now, it turns out that the general case of non-vanishing reflection, $B \neq 0$, can be reduced to (28) by a canonical Bogoliubov transformation of $c_{\alpha, \tau}$ making the quadratic form in (24) diagonal. The transformation is related in the usual way with the eigenvectors of the matrix $\widehat{W}$. Thus, we come to Eqs.(28), (29) with $\lambda$ replaced by $\lambda_{*}$ :

$$
\sin \frac{\lambda_{*}}{2}=|A| \sin \frac{\lambda}{2} .
$$

The counting statistics in this case are non-gaussian:

$$
\chi(\lambda)=e^{-\lambda_{*}^{2} f(t, T)} .
$$

One checks that the second moment of the distribution

$$
\left\langle\left\langle m^{2}\right\rangle\right\rangle=-\left.\frac{\partial^{2} \chi(\lambda)}{\partial \lambda^{2}}\right|_{\lambda=0}=2|A|^{2} f(t, T)
$$

agrees with the Johnson-Nyquist formula for the equilibrium noise. 


\section{STATISTICS OF A DC CURRENT: QUANTUM SHOT NOISE}

Let us consider non-equilibrium noise. In this case, due to the asymmetry in the distributions, $n_{L(R)}(E)=\left(\exp \left(E \pm \frac{1}{2} \mathrm{eV}\right) / T+1\right)^{-1}$, generally one cannot uncouple the two channels by a canonical transformation. We calculate the statistics within an approximation that ignores the effect of switching at $\tau=0$ and $\tau=t$. Let us close the axis $\tau$ into a circle of length $t$, which amounts to restricting on periodic states:

$$
\psi(\tau)=\psi(\tau \pm t)
$$

For the $t$-periodic problem, by going to the Fourier space, one has

$$
\begin{aligned}
\chi(\lambda)= & \prod_{k \in Z}\left[1+|A|^{2}\left(e^{-i \lambda}-1\right) n_{L}\left(E_{k}\right)\left(1-n_{R}\left(E_{k}\right)\right)\right. \\
& \left.+|A|^{2}\left(e^{i \lambda}-1\right) n_{R}\left(E_{k}\right)\left(1-n_{L}\left(E_{k}\right)\right)\right],
\end{aligned}
$$

where $E_{k}=2 \pi \hbar k / t, k$ is an integer. For large $t, t \gg \hbar / T$ or $t \gg \hbar / e V$, the product is converted to an integral:

$$
\begin{array}{r}
\ln (\chi(\lambda))=\frac{t}{2 \pi \hbar} \int_{-\infty}^{+\infty} d E \ln \left(1+|A|^{2}\left(e^{-i \lambda}-1\right)\right. \\
\left.\times n_{L}\left(1-n_{R}\right)+|A|^{2}\left(e^{i \lambda}-1\right) n_{R}\left(1-n_{L}\right)\right) .
\end{array}
$$

We evaluate it analytically, and get

$$
\chi(\lambda)=\exp \left(-t T u_{+} u_{-} / h\right),
$$

where

$$
u_{ \pm}=v \pm \cosh ^{-1}\left(|A|^{2} \cosh (v+i \lambda)+|B|^{2} \cosh v\right)
$$


$v=e V / 2 T$. The answer simplifies in the two limits: $T \gg e V$ and $e V \gg T$. In the first case we return to the equilibrium result (33). In the second case, corresponding to the recently discussed quantum shot noise [3] 5], we have

$$
\chi(\lambda)=\left(e^{i \epsilon \lambda}|A|^{2}+|B|^{2}\right)^{e|V| t / h}, \epsilon=\operatorname{sgn} V,
$$

Analyzed according to Eq.(3), this $\chi(\lambda)$ leads to the binomial distribution

$$
P_{N}(m)=p^{m} q^{N-m} C_{N}^{m},
$$

$p=|A|^{2}, q=|B|^{2}, N=e|V| t / h$. One checks that the moments $\langle m\rangle=p N$ and $\left\langle\left\langle m^{2}\right\rangle\right\rangle=p q N$ correspond directly to the Landauer formula and to the formula for the intensity of the quantum shot noise [3 5]. The correction to the statistics due to the switching effects is insignificant [10].

\section{NOISE DUE TO A VOLTAGE PULSE: ORTHOGONALITY CATASTROPHE}

Here we consider the fluctuations of current in a single-channel conductor induced by a voltage pulse. The result will be that the dependence of the fluctuations on Faraday's flux $\Phi=-c \int V(t) d t$ contains a logarithmically divergent term periodic in $\Phi$ with the period $\Phi_{0}=h c / e$. The fluctuation is smallest near $\Phi=n \Phi_{0}$. The divergence is explained by a comparison with the orthogonality catastrophe problem. The $\Phi_{0}$-periodicity is related with the discreteness of "attempts" in the binomial statistics picture of charge fluctuations presented above.

Initially, the orthogonality catastrophe problem emerged from the observation that the ground state of a Fermi system with a localized perturbation is orthogonal to the non- 
perturbed ground state, no matter how weak the perturbation [27]. Originally, the discussion was focused on the purely static effect of Fermi correlations on the ground state that leads to the orthogonality, but then it shifted to dynamical effects. When a sudden localized perturbation is turned on in a Fermi gas, the number of excited particle-hole pairs detected over a large time interval $t$ diverges as $\ln t / \tau$, where $\tau$ is the time of switching of the perturbation. This effect leads to power law singularities in transition rates involving collective response of fermions, such as X-ray absorption in metals [28,24]. In this section we present an application of the orthogonality catastrophe picture to the electric current noise.

Let us consider a single channel conductor in an external field described by the onedimensional Schrödinger equation

$$
\begin{aligned}
& i \frac{\partial}{\partial t} \psi(x, t)=\widehat{\mathcal{H}} \psi(x, t), \\
& \widehat{\mathcal{H}}=\frac{1}{2}\left(-i \frac{\partial}{\partial x}-\frac{e}{c} A(x, t)\right)^{2}+U(x),
\end{aligned}
$$

where the potential $U(x)$ represents the scattering region and $A(x, t)$ is the vector potential corresponding to the applied pulse of electric field. Since the pulse duration $\tau$ is assumed to be much longer than the time of scattering, one can treat the vector potential as static and apply a gauge transformation in order to accumulate the flux $\varphi(t)=e / \hbar \int_{-\infty}^{t} V\left(t^{\prime}\right) d t^{\prime}$ in the phases of the transmission amplitudes, thus making them time dependent. By going through the argument presented in Sec. IV, one obtains the scattering states (17) and (19) with time-dependent forward scattering amplitudes:

$$
A_{L(R)} \rightarrow A_{L(R)} e^{ \pm i \varphi\left(t_{r}\right)}
$$

where the time $t_{r}=t-|x| / v_{F}$ is taken retarded in order to account for the finite speed of motion after scattering. As before, here we assume that scattering by the potential as well 
as traversing the region where the voltage is applied takes negligible time compared to the duration of the voltage pulse. In this approximation the amplitudes of backward scattering $B_{L(R)}$ are time-independent constants.

To draw a relation with the orthogonality catastrophe problem, let us study the effect of the voltage pulse on the scattering phases $\delta_{1}, \delta_{2}$. They can be found by diagonalizing the scattering matrix

$$
\widehat{\mathcal{S}}(t)=\left[\begin{array}{cc}
A_{L} e^{i \varphi(t)+i \lambda / 2} & B_{R} \\
B_{L} & A_{R} e^{-i \varphi(t)-i \lambda / 2}
\end{array}\right],
$$

and writing its eigenvalues as $e^{i \delta_{1}}, e^{i \delta_{2}}$. The relation between the phases $\delta_{1,2}$ before and after the pulse is written conveniently through $\delta_{ \pm}=\left(\delta_{1} \pm \delta_{2}\right) / 2$. The phase $\delta_{+}$does not change at any time, and the phase $\delta_{-}$changes according to

$$
\cos ^{2} \delta_{-}\left(t^{\prime}\right)+\cos ^{2} \delta_{-}(t)-2 \cos \delta_{-}\left(t^{\prime}\right) \cos \delta_{-}(t) \cos \Delta \varphi=\left|A_{L}\right|^{2} \sin ^{2} \Delta \varphi,
$$

where $\Delta \varphi=\varphi\left(t^{\prime}\right)-\varphi(t)$. Now, let us compare to the orthogonality catastrophe in the Fermi system subjected to a time-dependent perturbation (43). Change of the flux induces the shift of the phases $\delta_{ \pm} \rightarrow \delta_{ \pm}^{\prime}$ and makes the new ground state orthogonal to the old one:

$$
\left\langle 0^{\prime} \mid 0\right\rangle=\exp \left(-2 \frac{\delta_{*}^{2}}{\pi^{2}} \ln \frac{L}{\lambda_{F}}\right),
$$

where $L$ is the system size, $\lambda_{F}$ is Fermi wavelength, and $e^{i \delta_{*}}$ is an eigenvalue of the matrix $\widehat{\mathcal{S}}^{-1}(t=\infty) \widehat{\mathcal{S}}(t=-\infty):$

$$
\sin \frac{\delta_{*}}{2}=\left|A_{L}\right| \sin \frac{\Delta \varphi}{2} .
$$

In terms of dynamics, this implies that the old ground state is shaken up so that infinitely many particle-hole pairs are excited [24]. It should lead to a logarithmically diverging contribution to noise, since for each of the particle-hole pairs there is a finite probability (equal to 
$\left.\left|A_{L} B_{R}\right|^{2}\right)$ that the particle and the hole will go to different terminals of the conductor, thus resulting in a current fluctuation. The periodicity in Faraday's flux $\Phi=-c \int V(t) d t$ follows from the gauge invariance and is explicit in Eqs.(44,46) for $\delta_{ \pm}^{\prime}$. The logarithmic divergence vanishes at $\Phi=n \Phi_{0}$, as expected, since at integer $\Phi$ there is no long-term change of the scattering.

Let us calculate the mean square fluctuation of the charge $\left\langle\left\langle Q^{2}\right\rangle\right\rangle$ transmitted through the system due to the pulse. For that, one can use the formula (26) with the time-dependent scattering matrix (43). To get the second cummulant $\left\langle\left\langle Q^{2}\right\rangle\right\rangle$ one expands the exponent (26) up to second order terms in $\lambda$, and takes an irreducible average using Wick theorem. The averages of $c_{i, \tau}$ have the usual form:

$$
\begin{aligned}
& \left\langle c_{i, \tau}^{+} c_{j, \tau^{\prime}}\right\rangle=\delta_{i j} \int n(E) e^{i E\left(\tau-\tau^{\prime}\right)} \frac{d E}{2 \pi}, \\
& \left\langle c_{i, \tau} c_{j, \tau^{\prime}}^{+}\right\rangle=\delta_{i j} \int(1-n(E)) e^{-i E\left(\tau-\tau^{\prime}\right)} \frac{d E}{2 \pi}
\end{aligned}
$$

where $n(E)=\left(e^{E / T}+1\right)^{-1}$ is the Fermi distribution. The result reads

$$
\begin{aligned}
\left\langle\left\langle Q^{2}\right\rangle\right\rangle= & \frac{\mathrm{g} e^{2}}{2 \pi} \int\left(|A|^{4}\left|\int_{0}^{t} e^{i \omega t^{\prime}} d t^{\prime}\right|^{2}+|A B|^{2}\right. \\
& \left.\times\left|\int_{0}^{t} e^{i \varphi\left(t^{\prime}\right)+i \omega t^{\prime}} d t^{\prime}\right|^{2}\right) \omega \operatorname{coth} \frac{\hbar \omega}{2 T} \frac{d \omega}{2 \pi}
\end{aligned}
$$

where $\mathrm{g}$ is spin degeneracy. The first term in (48) is a part of equilibrium noise since it does not depend on $\varphi$. To analyze the second term, let us take a step-like time dependence of $\varphi$ resulting from an abrupt voltage pulse applied at the time $t_{0}, 0<t_{0}<t$, the pulse duration $\tau$ being much shorter than $t$. Taking the integral and keeping only the terms diverging at $t \rightarrow \infty$, we find

$$
\frac{g e^{2}}{2 \pi} \int\left|\frac{e^{i \omega t_{0}}-1}{i \omega}+e^{2 \pi i \Phi / \Phi_{0}} \frac{e^{i \omega t}-e^{i \omega t_{0}}}{i \omega}\right|^{2}|\omega| \frac{d \omega}{2 \pi}
$$




$$
=\frac{g e^{2}}{\pi^{2}}\left(\ln \frac{t E_{F}}{\hbar}+2 \sin ^{2} \frac{\pi \Phi}{\Phi_{0}} \ln \frac{t}{\tau}\right)
$$

where the ultraviolet-diveregent integrals are cut at frequency $\sim E_{F} / \hbar$. By subtracting the result for $\Phi=0$ as corresponding to equilibrium, one obtains a logarithmic contribution to the non-equilibrium noise:

$$
\left\langle\left\langle Q^{2}\right\rangle\right\rangle=g e^{2}|A B|^{2}\left[\frac{2}{\pi^{2}} \sin ^{2} \frac{\pi \Phi}{\Phi_{0}} \ln \frac{t_{0}}{\tau}+\frac{\Phi}{\Phi_{0}}\right]+\ldots+\langle\langle Q\rangle\rangle_{e q}
$$

The origin of the non-diverging term in Eq. (50) proportional to $\Phi / \Phi_{0}$ will be discussed below. The dots in Eq. (50) represent corrections higher order in $\Phi_{0} / \Phi$, and the equilibrium noise

$$
\left\langle\left\langle Q^{2}\right\rangle\right\rangle_{e q}=\frac{e^{2} G}{\pi^{2}} \ln \frac{t E_{F}}{\hbar}, G=\mathrm{g} \frac{e^{2}}{\hbar}|A|^{2}
$$

is obtained by repeating the calculation for $\Phi=0$. The expression (51) agrees with the Nyquist formula

$$
\left\langle\left\langle j_{\omega} j_{-\omega}\right\rangle\right\rangle=e^{2} G \omega \operatorname{coth} \frac{\omega}{2 T}
$$

taken at $T=0$, Fourier transformed, and combined with the relation $Q=\int_{0}^{t} j\left(t^{\prime}\right) d t^{\prime}$.

The term in Eq. (50) proportional to $\Phi / \Phi_{0}$ is obtained by rewriting the integral in the second term of $(48)$ as

$$
\iiint \frac{d \omega}{2 \pi}|\omega| d t_{1} d t_{2} e^{i\left(\varphi\left(t_{1}\right)-\varphi\left(t_{2}\right)+\omega\left(t_{1}-t_{2}\right)\right)}
$$

and extracting the contribution of almost coinciding times $t_{1}$ and $t_{2}$ by going to new variables $t=\left(t_{1}+t_{2}\right) / 2, t^{\prime}=t_{1}-t_{2}$, and changing the order of integrations:

$$
\int d t \int \frac{d \omega}{2 \pi}|\omega| \int d t^{\prime} e^{i \varphi\left(t_{1}\right)-i \varphi\left(t_{2}\right)+i \omega t^{\prime}}=\int|\dot{\varphi}| d t
$$


where we replaced $\varphi\left(t_{1}\right)-\varphi\left(t_{2}\right)=\varphi\left(t+t^{\prime} / 2\right)-\varphi\left(t-t^{\prime} / 2\right)$ by $\dot{\varphi} t^{\prime}$. The result (54) is approximate: it does not give the log-term because the transformation (54) properly takes care of the integral (53) only in the domain $t_{1} \simeq t_{2}$, under the restriction that $\Phi(t)$ is varying sufficiently smoothly. When $\Phi(t)$ is a monotonous function, $\dot{\varphi}>0$, the integral in the right hand side of (54) equals $2 \pi \Phi / \Phi_{0}$ and thus produces the term of Eq. (50) proportional to $\Phi / \Phi_{0}$

It is clear from the derivation that the two terms in the brackets in Eq. (50) arise from different integration domains in the $t_{1}-t_{2}$ space: the first term corresponds to $\left|t_{1,2}\right| \geq \tau, t_{1} t_{2}<$ 0 , while the second one is due to almost coinciding moments, $\left|t_{1}-t_{2}\right| \ll \tau$. Since these domains are almost non-overlapping, the two contributions to the noise (50) do not interfere (cross terms are small).

In order to estimate the correction to the result (50), let us derive it by another method that allows to trace out the higher order terms. For that, let us take the flux in the form $\varphi(t)=N \lambda(t)$, where $\lambda(t)$ is a smooth monotonous function, $\lambda(-\infty)=0, \lambda(\infty)=2 \pi$. For integer $N \gg 1$ the Fourier component of $e^{i N \lambda(t)}$ entering Eq. (54) in the stationary phase approximation is given by

$$
\int_{-\infty}^{\infty} e^{i N \lambda(t)+i \omega t} d t=\sum_{k} \sqrt{\frac{2 \pi i}{N \ddot{\lambda}\left(t_{k}\right)}} e^{i N \lambda\left(t_{k}\right)+i \omega t_{k}}+\ldots
$$

where the dots indicate terms $\sim \mathrm{O}\left(N^{-3 / 2}\right)$, and $t_{k}$ 's are real solutions of the equation $N \dot{\lambda}(t)+\omega=0$. Then we can write

$$
\left|\int_{-\infty}^{\infty} e^{i N \lambda(t)+i \omega t} d t\right|^{2}=\sum_{k} \frac{2 \pi}{N \ddot{\lambda}\left(t_{k}\right)}+\mathrm{O}\left(N^{-2}\right)
$$

and thus obtain

$$
\left\langle\left\langle Q^{2}\right\rangle\right\rangle=A \int_{-\infty}^{\infty} \sum_{k} \frac{|\omega| d \omega}{N \ddot{\lambda}\left(t_{k}\right)}+\ldots
$$


where the dots represent higher order terms. By differentiating both sides of the equation $N \dot{\lambda}(t)=-\omega$ one finds the relation $d \omega=-N \ddot{\lambda}\left(t_{k}\right) d t_{k}$, which means that $|\omega| d \omega / \ddot{\lambda}\left(t_{k}\right)=$ $-\left|\dot{\lambda}\left(t_{k}\right)\right| d t_{k}$, and therefore the integral in Eq. (57) equals $N \int_{-\infty}^{\infty} d \lambda=2 \pi N$. Since $|\omega| d \omega$ scales as $N^{2}$, the correction to Eq. (57) can be evaluated as $\mathrm{O}(1)$, i.e., it is of the order of one for any $N$. This means that Eq. (77) has relative accuracy of $\mathrm{O}(1 / N)$.

The term in (50) proportional to $\Phi / \Phi_{0}$ is interesting in connection with the picture of binomial statistics presented in Sec.VI. In the dc bias case, the distribution of charge for a single channel situation was found to be binomial with frequency of attempts equal to $\mathrm{eV} / \mathrm{h}$ and the probabilities of outcomes $p=|A|^{2}, q=|B|^{2}$. Taken literally, this means that the attempts to transfer charge are repeated regularly in time, almost periodic with the period $h / e V$, with each attempt having two outcomes - transmission or reflection - occurring with the probabilities $p$ and $q$. However, the regularity of the attempts does not lead to an ac component in the current, rather it appears just as a part of statistical description of charge fluctuations. Still, the presence of a non-zero frequency in a non-interacting system requires interpretation.

Let us suppose that the flux varies linearly with time, $\Phi(t)=-c V t$. Since the e.m.f. $=$ $-\partial \Phi / c \partial t$, the linear dependence of $\Phi(t)$ is equivalent in its effect on the noise to constant voltage $V$. In accordance with one's expectation, the second term in the brackets in Eq. (50) for a single channel is $\left\langle\left\langle Q^{2}\right\rangle\right\rangle=\mathrm{g} e^{2}|A B|^{2} \Phi / \Phi_{0}$, i.e., it is precisely of the form arising from the binomial distribution with probabilities of outcomes $p$ and $q$, and the number of attempts $N=\Phi / \Phi_{0}$. (Let us recall that the second moment of the binomial distribution equals $p q N$.) Taking into account that the time during which the flux changes by $\Phi_{0}$ is $h / e V$, we can interpret the number of attempts in the statistical picture as the number of flux quanta by 
which the flux is changed. Such a conclusion suggests an interesting generalization of the picture of binomial statistics by attributing the meaning of the number of attempts to the

flux change measured in the units of $\Phi_{0}$, regardless of the linear or non-linear character of the flux dependence on time.

It is appealing to put the special role of integer fluxes in connection with the binomial statistics of current, where the flux quanta are naturally interpreted as discrete attempts to transmit charge. Although this picture is yet to be confirmed by analytic treatment, it receives some support from the property of the $\Phi_{0}$-periodic term in (50) to vanish at every integer $\Phi$. One may conjecture that the statistics are close to binomial only when the flux change is an integer, and have diverging logarithmic corrections otherwise. The distinction that Eq. (50) makes between integer and non-integer values of the flux and the relation of integer flux change to the number of attempts in the binomial distribution, gives another perspective to the statistical picture of a current pulse.

To summarize, the fluctuations caused by a voltage pulse, in contrast to the average transmitted charge, distinguish between integer and non-integer flux change. As a result, the dependence of noise on the flux is non-monotonous and has minima at integer values of the flux.

\section{COHERENT STATES OF CURRENT}

The question we address in this section is about optimal way of changing flux that minimizes induced noise. It is clear from what has been said that to achieve minimum of the noise one should change the flux by an integer amount, 


$$
\Delta \varphi=\varphi(t=\infty)-\varphi(t=-\infty)=2 \pi n
$$

in order to suppress the logarithmically divergent term. However, since for a given $\Delta \varphi$ the noise depends on the actual function $\varphi(t)$, not just on $\Delta \varphi$, we have a variational problem to solve for the noise as a functional of the time dependence of the flux. This functional was derived in Sec. VII. At zero temperature it is given by

$$
\left\langle\left\langle Q^{2}\right\rangle\right\rangle=\frac{\mathrm{g} e^{2}}{2 \pi}|A B|^{2} \int\left|\int e^{i \varphi(t)+i \omega t} d t\right|^{2}|\omega| \frac{d \omega}{2 \pi}
$$

where $A$ and $B$ are transmission and reflection amplitudes, and $\mathrm{g}$ is spin degeneracy. We shall study the variational problem (49) with the boundary condition (58) and show that its general solution has the form of a sum of soliton-like functions:

$$
\Phi(t)= \pm \frac{\Phi_{0}}{\pi} \sum_{k=1}^{n} \tan ^{-1}\left(\frac{t-t_{k}}{\tau_{k}}\right), \tau_{k}>0
$$

where $t_{k}$ and $\tau_{k}$ are arbitrary constants. Under the condition (58), any time dependence of the form (60) gives absolute minimum to the noise:

$$
\min \left[\left\langle\left\langle Q^{2}\right\rangle\right\rangle\right]=g e^{2}|A B|^{2}|n|
$$

For an optimal time dependence of the voltage $V=-\partial \Phi / c \partial t$, therefore, one has a sum of Lorentzian peaks:

$$
V(t)=\mp \frac{\Phi_{0}}{c \pi} \sum_{k=1}^{n} \frac{\tau_{k}}{\left(t-t_{k}\right)^{2}+\tau_{k}^{2}}
$$

In order to compare quantum noise with conductance, let us mention that the average transmitted charge

$$
\langle\langle Q\rangle\rangle=\mathrm{g} e|A|^{2} \frac{\Delta \varphi}{2 \pi}=\mathrm{g} \frac{e^{2}}{h}|A|^{2} \int V(t) d t
$$


simply obeys the Ohm's law, i.e., there is no particular dependence on the way the flux change $\Delta \varphi$ is realized.

The result (60), 61] has a simple interpretation in terms of the binomial statistics picture of charge fluctuations. For the binomial distribution with probabilities of outcomes $p$ and $q, p+q=1$, and with the number of attempts $N$, the second moment is known to be equal to $p q N$. The comparison with Eq. (61) suggests to attribute to $n=\Delta \Phi / \Phi_{0}$ the meaning of the number of attempts. This interpretation is supported by the structure of the function (60) consisting of $n$ terms, each corresponding to unit change of flux. A remarkable property of the function (60) is its separability, manifest both in the form of the terms and in the way the parameters $t_{k}, \tau_{k}$ enter the expression. Let us note that by making some of the $t_{k}$ 's close to each other one can have an overlap in time of different "attempts". The overlap, however, does not change the fluctuations (61). The situation reminds the one with solitons in integrable non-linear systems, or with non-interacting instantons in integrable field theories. Also, the absence of interference is interesting in the context of coherent nature of transport in this system: after all, we simply have scattering by a time-dependent potential. Perhaps, proper interpretation of this effect should be sought in establishing relation with the theory of coherent states, known to eliminate to some extent the quantum mechanical interference.

Let us now turn to the variational problem. It is convenient to do the integral over $\omega$ first and to rewrite (49) as

$$
\left\langle\left\langle Q^{2}\right\rangle\right\rangle=-\frac{D}{\pi} \iint \frac{e^{i \varphi(t)-i \varphi\left(t^{\prime}\right)}}{\left(t-t^{\prime}\right)^{2}} d t d t^{\prime}
$$

where $D=\frac{\mathrm{g} e^{2}}{2 \pi}|A B|^{2}$. In order to avoid divergence at $t=t^{\prime}$ the denominator in (64) should 
be understood as

$$
\frac{1}{2}\left[\frac{1}{\left(t-t^{\prime}+i \delta\right)^{2}}+\frac{1}{\left(t-t^{\prime}-i \delta\right)^{2}}\right], \quad \delta \rightarrow 0
$$

the condition that one obtains by introducing regularization in (49): $|\omega| \rightarrow|\omega| e^{-|\omega| \delta}$. By considering variation of the functional (64) we have the equation for an extremum:

$$
\operatorname{Im}\left[e^{i \varphi(t)} \int \frac{e^{-i \varphi\left(t^{\prime}\right)}}{\left(t-t^{\prime}\right)^{2}} d t\right]=0
$$

By using Cauchy formula one checks that the functions

$$
e^{i \varphi(t)}=\prod_{k=1}^{n} \frac{t-\lambda_{k}}{t-\bar{\lambda}_{k}}, \lambda_{k}=t_{k}+i \tau_{k}
$$

satisfy (66) provided that $\tau_{k}$ 's are all of the same sign. Obviously, the functions (67) are just another form of $(60)$.

It remains to be shown that the functional reaches its minimum on the solutions (67). To prove it we proceed in the following steps. Let us write $e^{i \varphi(t)}$ as

$$
e^{i \varphi(t)}=f_{+}(t)+f_{-}(t)
$$

where $f_{+}(t)$ and $f_{-}(t)$ are bounded analytic functions in the upper and lower complex $t$ halfplane, respectively. Representation (68) exists for any non-singular function and defines the functions $f_{+}$and $f_{-}$up to a constant. Then we substitute Eq. (68) in (64), and apply Cauchy formula for the derivative,

$$
\dot{f}_{ \pm}(t)= \pm \frac{i}{2 \pi} \oint \frac{f_{ \pm}\left(t^{\prime}\right) d t^{\prime}}{\left(t-t^{\prime} \pm i 0\right)^{2}}
$$

where the contour of integration is chosen in the half-plane of analyticity of $f_{+}$or $f_{-}$, respectively. Thus one gets 


$$
\left\langle\left\langle Q^{2}\right\rangle\right\rangle=-i D \int\left(\bar{f}_{+} \dot{f}_{+}-\bar{f}_{-} \dot{f}_{-}\right) d t
$$

On the other hand,

$$
\begin{aligned}
n & =\frac{1}{2 \pi i} \int e^{-i \varphi(t)} \frac{d}{d t} e^{i \varphi(t)} d t \\
& =-\frac{i}{2 \pi} \int\left(\bar{f}_{+} \dot{f}_{+}+\bar{f}_{-} \dot{f}_{-}\right) d t
\end{aligned}
$$

where the last equality is a result of substituting (68) and using the relations

$$
\int \bar{f}_{+} \dot{f}_{-}=\int \bar{f}_{-} \dot{f}_{+}=0
$$

which follow from Cauchy theorem. Now, Eq. (70) can be rewritten through Fourier components of $f_{+}$and $f_{-}$as

$$
\left\langle\left\langle Q^{2}\right\rangle\right\rangle=D \int_{0}^{\infty}\left(\left|f_{+}(\omega)\right|^{2}+\left|f_{-}(-\omega)\right|^{2}\right) \omega \frac{d \omega}{2 \pi}
$$

thus demonstrating positivity of both terms in (70). (It is used that $f_{+}(\omega)=f_{-}(-\omega)=0$ for $\omega<0$.) With this, by comparing $(70)$ and $(71)$ we obtain

$$
\left\langle\left\langle Q^{2}\right\rangle\right\rangle \geq 2 \pi D|n|
$$

Equality in (74) is reached only when either $f_{+}(t)$ or $f_{-}(t)$ vanishes. Therefore, to obtain the minimum one has to take the functions $e^{i \varphi(t)}$ that are regular in one of the half-planes. This remark is sufficient to see that the functions (67) form a complete family of solutions.

It is worth mentioning that the method used to derive (74) copies almost entirely the procedure of derivation of the duality condition in the theory of instantons. Like in other situations where the duality condition holds, our "solitons" do not interact: $\left\langle\left\langle Q^{2}\right\rangle\right\rangle$ shows no dependence on the parameters $\lambda_{k}$ of the solution (67). Among numerous field theories that allow for exact solution of the instanton problem the one most similar to our case is 
the theory of classical Heisenberg ferromagnet in two dimensions. For this problem the instantons were found by mapping the order parameter space (i.e., the unit sphere) on the complex plane [29]. The duality condition was shown to take the form of the constraint of analyticity or anti-analyticity of the mapped order parameter function (compare with the condition $f_{+}=0$ or $f_{-}=0$ derived above). Multi-instanton solutions were given as products of single instanton solutions (cf. Eq. (67)). This analogy obviously deserves more attention.

At this point let us examin an interesting non-optimal time dependence of the flux, the sum of two solitons with opposite charge:

$$
\varphi(t)=2\left[\tan ^{-1}\left(\frac{t-t_{1}}{\tau_{1}}\right)-\tan ^{-1}\left(\frac{t-t_{2}}{\tau_{2}}\right)\right]
$$

$\tau_{1,2}>0$. This function corresponds to $e^{i \varphi(t)}$ of the form (67) but with the poles in both half-planes. In this case $\Delta \varphi=0$, and thus $\langle\langle Q\rangle\rangle=0$, so $\min \left[\left\langle\left\langle Q^{2}\right\rangle\right\rangle\right]=0$. With the function (75), however, one finds

$$
\left\langle\left\langle Q^{2}\right\rangle\right\rangle=4 \pi D\left|\frac{\lambda_{1}-\lambda_{2}}{\lambda_{1}-\bar{\lambda}_{2}}\right|^{2}
$$

where $\lambda_{1,2}=t_{1,2}+i \tau_{1,2}$. For different values of the parameters $t_{1,2}, \tau_{1,2}$ Eq. (76) interpolates between two trivial limiting cases:

(i) $\left\langle\left\langle Q^{2}\right\rangle\right\rangle \rightarrow 0$, when the two flux steps in (75) have nearly the same duration and almost overlap;

(ii) $\left\langle\left\langle Q^{2}\right\rangle\right\rangle \rightarrow 4 \pi A$, when the flux steps either differ strongly in their duration or do not overlap.

In the case (ii) the noise is twice bigger than the noise due to a single step, as it should be.

We see that when $\Delta \varphi / 2 \pi$ is of the order of one a non-optimal time dependence $\varphi(t)$ can considerably enhance the noise. It is not the case, however, for $\Delta \varphi / 2 \pi \gg 1$. This limit was 
studied in Sec. VII, where it was found that when $\varphi(t)$ is a monotonous function the result

$$
\left\langle\left\langle Q^{2}\right\rangle\right\rangle=\mathrm{g} e^{2}|A B|^{2}|\Delta \varphi / 2 \pi|
$$

is rather accurate, even if the time dependence $\varphi(t)$ is not optimal [13].

A more intuitive way to understand the accuracy of Eq. (77) is to note that for a given $n$ the number of parameters in the optimal flux dependence (53) is $2 n$, which means that half of them are in some sense redundant. Because of that any smooth monotonous function with sufficiently large variation $\Delta \varphi$ can be rather accurately approximated by a function of the form (53), and therefore the noise exceeds the lower bound just slightly.

An implication of this result for the binomial statistics picture is as follows. As it was discussed above there is a (conjectured) correspondence of the terms of Eq. (60) and of the attempts. The deviation from the binomial distribution, that of course should exist for a non-optimal flux function $\varphi(t)$, will remain bounded in the case of a smooth $\varphi(t)$, as $\Delta \varphi$ increases taking integer values. More precisely, the distribution will be written as a mixture of binomial distributions with different numbers $N$ of attempts, $P(m)=\sum_{N} \rho_{N} P_{N}(m)$, where $P_{N}(m)=p^{m} q^{N-m} C_{N}^{m}$. The estimated correction implies that the distribution of attempts $\rho_{N}$ has finite variance in the limit $N=\Delta \varphi / 2 \pi \rightarrow \infty$.

Before closing, let us mention that in order to apply the results of Secs. VII, VIII to transport in a mesoscopic metallic conductor with disorder, described by many conducting channels with transmission constants $T_{n}$, one just needs to replace $|A B|^{2}$ by $\sum_{n} T_{n}\left(1-T_{n}\right)$, since different scattering channels contribute to the noise independently. The condition of validity of our treatment then is that the variation of the flux is sufficiently slow, so that $\min \left[\tau_{k}\right] \gg \hbar / E_{c}$, the time of diffusion across the sample. However, at non-zero tempera- 
ture one also has to satisfy the condition $\tau_{k} \ll \hbar / T$, the time of phase breaking. So, the temperature interval where our estimate of the noise holds is $T \leq E_{c}$.

\section{CONCLUSIONS}

We introduced a quantum-mechanical scheme that gives complete statistical description of electron transport. It involves a spin $1 / 2$ coupled to the current so that the spin precession measures transmitted charge. The off-diagonal part of the spin density matrix, taken as a function of the coupling constant, gives the generating function for the electron counting statistics. We find the statistics in a single-channel ideal conductor for arbitrary relation between temperature and voltage. In equilibrium, the counting statistics are gaussian, both for zero-point fluctuations and at finite temperature. At constant voltage and low temperature the statistics are Bernoullian and the distribution is binomial.

The theory leads to interesting conclusions applied to the current fluctuations produced by a voltage pulse. In this case, the noise has phase sensitivity: it oscillates as function of Faraday's flux, $c \int V(t) d t$, reaching minimum at integer fluxes. We studied the noise as function of the shape of the voltage pulse and found optimal time dependence that provides absolute minimum of the noise for given average transmitted charge. Solution displays interesting analogy with the problem of instantons in the field theories obeying duality symmetry. Optimal time dependence is a sum of Lorentzian peaks of voltage, each corresponing to a soliton of flux. The change of flux for a soliton is equal to the flux

quantum $\Phi_{0}$. The solitons are interpreted in terms of the binomial statistics picture of charge fluctuations as attempts to transmit electrons, one electron per soliton. 


\section{APPENDIX A: LARMOR CLOCK MEASUREMENT OF TUNNELING TIME}

How long does it take a particle to tunnel under a barrier? More precisely, suppose a particle of energy $E$ is moving in one dimension, and is scatterred on a potential barrier:

$$
i \frac{\partial}{\partial t} \psi(x, t)=\left[-\frac{1}{2} \frac{\partial^{2}}{\partial x^{2}}+U(x)\right] \psi(x, t) .
$$

What is the probability that during the scattering the particle spends time $\tau$ within the region $a<x<b$ under the barrier? Questions of that kind arise naturally in discussion of any quantum-mechanical process that takes finite time, like nuclear or chemical reactions, resonance scattering, or tunneling.

There have been several attempts to treat such problems 23 that resulted in formulation of a very interesting concept of Larmor clock. It has various analogies with the spin galvanometer discussed above, and it seems useful to review the Larmor clock here using the same language. The Larmor clock uses an auxiliary spin 1/2 attached to the scattering particle, and an auxiliary constant magnetic field $\omega$ localized within the region of interest, $a<x<b$,

$$
\widehat{\mathcal{H}}_{\text {int }}=-\frac{1}{2} \omega \sigma_{z} \int_{a}^{b} \psi^{+}(x) \psi(x) d x .
$$

The choice of coupling is such that the spin precession angle is proportional to the time spent in the region $a<x<b$. The difference from our spin-galvanometer is that the spin is not stationary, but travels with the particle, and also that the spin is coupled to the particle density, rather than to the current.

To find the distribution of times one has to write down the system density matrix evolved in time, and take partial trace over the particle outgoing states. (We assume that one 
does not have to distinguish between different results of scattering, and is interested in the tunneling time only, regardless of whether the particle went through the barrier, or has been reflected.) Then, by following the argument of Sec. III one obtains the spin density matrix:

$$
\widehat{\rho}_{s}(t)=\left[\begin{array}{cc}
\hat{\rho}_{\uparrow \uparrow}(0) & \chi(\omega) \widehat{\rho}_{\uparrow \downarrow}(0) \\
\chi(-\omega) \hat{\rho}_{\downarrow \uparrow}(0) & \widehat{\rho}_{\downarrow \downarrow}(0)
\end{array}\right] .
$$

Here

$$
\chi(\omega)=\operatorname{tr}_{e}\left(e^{-i \widehat{\mathcal{H}}_{\omega} t} \widehat{\rho}_{e} e^{i \widehat{\mathcal{H}}_{-\omega} t}\right)
$$

where $e^{-i \widehat{\mathcal{H}}_{\omega} t}$ is the evolution operator for the one-particle problem with no spin:

$$
i \frac{\partial}{\partial t} \psi(x, t)=\left[-\frac{1}{2} \frac{\partial^{2}}{\partial x^{2}}+U(x)-\frac{1}{2} \omega \theta_{a b}(x)\right] \psi(x, t)
$$

where $\theta_{a b}=\theta(x-a) \theta(b-x)$. The auxiliary magnetic field $\omega$ now turns into a constant potential within the region $a<x<b$. Here again, with the spin degrees of freedom taken care of by (A3), we are left with a single particle problem. By using cyclic property of the trace one finds

$$
\chi(\omega)=\left\langle e^{i \widehat{\mathcal{H}}_{-\omega} t} e^{-i \widehat{\mathcal{H}}_{\omega} t}\right\rangle
$$

Here the brackets $\langle\ldots\rangle$ mean averaging over the particle initial state. Note that $\chi(\omega)$ is written in terms of a purely single particle problem, not involving spin variables.

The quantity $\chi(\omega)$ obtained by measuring precession of the spin is a generating function for the distribution of times, which is clear from the Fourier transform

$$
\chi(\omega)=\int P(\tau) e^{i \omega \tau} d \tau
$$

The probabilities $P(\tau)$ of different precession angles of the spin should be interpreted as the scattering time distribution. 
The probabilities $P(\tau)$ defined by (A5), (A6), and (A7) have several interesting properties:

a) $\int P(\tau) d \tau=1$;

b) $P(\tau)$ are real numbers;

c) $P(\tau)$ vanish at negative times $\tau<0$.

The normalization property $a$ ) is derived from (A6) by setting $\omega=0$. Property $b$ ) (real valuedness) is derived from $\chi(-\omega)=\bar{\chi}(\omega)$ which follows from (A6). The causality property c) follows from considering the evolution in the problem (A5) with $\omega$ continued to complex values. One notes that both the solution $\psi(x, t)$ of Eq. (A5) and the evolution operator $e^{-i \widehat{\mathcal{H}}_{\omega} t}$ are regular in the upper half-plane $\operatorname{Im} \omega>0$, which means that the same is true for $\chi(\omega)$. From that, the causality property $c$ ) follows by the usual argument using Cauchy theorem in the integral

$$
P(\tau)=\int_{-\infty}^{\infty} \chi(\omega) e^{-i \omega \tau} \frac{d \omega}{2 \pi}
$$

by closing the integration contour in the upper half-plane.

The properties $a$ ), b) and $c$ ) suggest that $P(\tau)$, so far defined formally as Fourier spectrum of $\chi(\omega)$, can have a meaning of probability. However, generally the sign of $P(\tau)$ can be either positive or negative, which makes the probabilistic interpretation problematic.

For the one particle problem one can write the generating function $\chi(\omega)$ in terms of the scattering amplitudes $A$ and $B$. For that, it is convenient to use the expressions (23), (24) for the evolution operator in terms of the scattering matrix $\widehat{\mathcal{S}}$, written using the wave-packet scattering states (19). Specializing to one particle and taking partial trace, one finds

$$
\chi(\omega)=\bar{A}_{-\omega}(E) A_{\omega}(E)+\bar{B}_{-\omega}(E) B_{\omega}(E),
$$


where $A(\omega)$ and $B(\omega)$ are the transmission and reflection amplitudes of the problem (A5) taken at the energy $E$ of incident particle.

To see the Larmor clock working, let us consider an example of resonance scattering, where a particle is scattered on a potential forming a quasibound state of life-time $\Gamma$. Using the method described above one can find the distribution of times it takes the particle to scatter. For simplicity, suppose that the particle can be only reflected, but not transmitted $(A=0)$. Then the reflection amplitude as function of energy is given by the Breit-Wigner formula:

$$
B(E)=\frac{E-E_{0}-i \Gamma / 2}{E-E_{0}+i \Gamma / 2} .
$$

Turning on the field $\omega$ in the quasibound state region is equivalent to shifting the resonance energy: $E_{0} \rightarrow E_{0}-\omega / 2$. Thus, the generating function of the time distribution is

$$
\chi(\omega)=\frac{\varepsilon-\omega+i \Gamma}{\varepsilon-\omega-i \Gamma} \frac{\varepsilon+\omega-i \Gamma}{\varepsilon+\omega+i \Gamma},
$$

where $\varepsilon=2\left(E-E_{0}\right)$. The distribution $P(\tau)$ is found by Fourier transform:

$$
\begin{aligned}
P(\tau) & =\int \chi(\omega) e^{-i \omega \tau} \frac{d \omega}{2 \pi} \\
& =\delta(\tau)-\frac{4 \Gamma}{\varepsilon}(\Gamma \sin \varepsilon \tau-\varepsilon \cos \varepsilon \tau) e^{-\Gamma \tau} \\
& =\frac{\partial}{\partial \tau}\left(\theta(\tau)-\frac{4 \Gamma}{\varepsilon} \sin \varepsilon \tau e^{-\Gamma \tau}\right) .
\end{aligned}
$$

The $\delta$-function term corresponds to the non-resonance scattering channel. Other terms describe dwelling in the quasibound state. In this example $P(\tau)$ is changing sign, which makes the probabilistic interpretation ambiguous.

The paradox arising due to negative $P(\tau)$ is only an apparent one. Really, the measurement of time performed by the Larmor clock is not the usual quantum-mechanical measurement, since the time is not an operator, and thus it cannot be measured in the same sense 
as other quantum-mechanical observables. This should be contrasted with the measurement of charge described above. Although the spin precession measurement scheme we use looks quite similar to the Larmor clock, there is a difference: Electric charge is an observable in the usual quantum-mechanical sense, it takes quantized integer values, and the probabilities of those values resulting from our calculation are non-negative.

\section{APPENDIX B: BOSONIZATION CALCULATION OF COUNTING STATISTICS}

In order to find generating function of counting statistics for a single channel conductor, we have to evaluate

$$
\chi(\lambda)=\left\langle\exp i \lambda \widehat{N}_{t}\right\rangle
$$

where $\widehat{N}_{t}=\int\left(c_{1, \tau}^{+} c_{1, \tau} c_{2, \tau}^{+} c_{2, \tau}\right) d \tau$, and $c_{i, \tau}, c_{i, \tau}^{+}$are canonical Fermi operators.

In one dimension, there is an equivalence between ideal Fermi gas and harmonic Bose chain, which provides a representation of the Fermi problem in terms of free bosons, known as the bosonization transformation [24 26]. This representation facilitates calculting averages like $(\mathbb{B 1})$, since they are being transformed to the form of a gaussian average [25].

According to the bosonization theory, bosonic Hamiltonian representing the fermionic problem is written as

$$
\widehat{\mathcal{H}}_{\text {Bose }}=\frac{\hbar v_{F}}{4 \pi} \int:\left(\nabla \theta_{L}\right)^{2}:+:\left(\nabla \theta_{R}\right)^{2}: d x
$$

where $\theta_{L(R)}(x)$ are Bose operators,

$$
\left[\nabla \theta_{L(R)}(x), \theta_{L(R)}(y)\right]= \pm 2 \pi i \delta(x-y)
$$


Connection to the fermionic problem is given as a relation between the densities of the leftand right-moving fermions, $\widehat{\rho}_{i}(x)=c_{i, x}^{+} c_{i, x}, i=1,2$, and the bosonic variables $\theta_{L(R)}(x)$, written as

$$
\widehat{\rho}_{1(2)}(x)=\frac{1}{2 \pi} \nabla \theta_{L(R)}(x)
$$

One notes that the operator $\widehat{N}_{t}$ in (B1) is linear in the densities $\widehat{\rho}_{i}$, and thus it is represented by an expression linear in the bosonic variables,

$$
\widehat{N}_{t}=\frac{1}{2 \pi}\left(\theta_{L}(t)-\theta_{L}(0)-\theta_{R}(t)+\theta_{R}(0)\right)
$$

which turns the average in (B1) into a gaussian type.

Therefore, the average of (28) is equal to the product of averages

$$
\chi(\lambda)=\left\langle\exp \frac{i \lambda}{2 \pi}\left(\theta_{L}(t)-\theta_{L}(0)\right)\right\rangle\left\langle\exp \frac{-i \lambda}{2 \pi}\left(\theta_{R}(t)-\theta_{R}(0)\right)\right\rangle
$$

taken over the ground state of the Hamiltonian (B2). To perform the average in (B6), it is sufficient to deal with the average over $\theta_{L}$ 's, because of the left-right symmetry of the problem.

Let us write $\theta_{L}(x)$ in terms of bosonic operators of plane waves:

$$
\begin{aligned}
\theta_{L}(x) & =\sum_{k>0}\left(\frac{2 \pi}{k}\right)^{1 / 2}\left[e^{i k x} b_{k}+e^{-i k x} b_{k}^{+}\right] \\
\nabla \theta_{L}(x) & =\sum_{k>0}(2 \pi k)^{1 / 2} i\left[e^{i k x} b_{k}-e^{-i k x} b_{k}^{+}\right] .
\end{aligned}
$$

One checks that the commutation relations (B3) are consistent with canonical commutation relations between $b_{k}$ and $b_{k^{\prime}}^{+}$. (The Hamiltonian of left-moving fermions is represented by $\widehat{\mathcal{H}}_{L}=\sum_{k>0} v k b_{k}^{+} b_{k}$.) The quantity $\theta_{L}(t)-\theta_{L}(0)$ appearing in the average (B6) is written as

$$
\sum_{k>0}\left(\frac{2 \pi}{k}\right)^{1 / 2}\left[\left(e^{i k v t}-1\right) b_{k}+\left(e^{-i k v t}-1\right) b_{k}^{+}\right] .
$$


We evaluate the average

$$
\begin{aligned}
& \left\langle\left\langle\left(\theta_{L}(t)-\theta_{L}(0)\right)^{2}\right\rangle\right\rangle=\sum_{k>0} \frac{2 \pi}{k}\left|e^{i k v t}-1\right|^{2}\left(2 N_{\text {Bose }}(k v / T)+1\right) \\
& =4 \int_{-\infty}^{\infty} \frac{d k}{|k|} \sin ^{2}(v k t / 2) \operatorname{coth}(v k / 2 T)=2 \ln \left(\frac{1}{\pi T \delta} \sinh (\pi T t)\right) .
\end{aligned}
$$

This expression equals $(2 \pi)^{2}$ times the function $f(t, T)$ computed in $(\sqrt{30})$. From that, we find the average $(\mathbb{B} 6)$ to be

$$
\begin{aligned}
& \chi(\lambda)=\left\langle\exp \frac{i \lambda}{2 \pi}\left(\theta_{L}(t)-\theta_{L}(0)\right)\right\rangle^{2} \\
& =\exp \left[-\left(\frac{\lambda}{2 \pi}\right)^{2}\left\langle\left\langle\left(\theta_{L}(t)-\theta_{L}(0)\right)^{2}\right\rangle\right\rangle\right]=\exp \left(-\lambda^{2} f(t, T)\right)
\end{aligned}
$$

which is the desired result.

Periodicity of $\chi(\lambda)$ in $\lambda$, corresponding to the charge quantization, is recovered if one corrects the relation between $\widehat{\rho}_{i}(x)$ and $\theta_{i}(x)$, in order to take into account the integervaluedness of the particle number $\widehat{N}_{t}$. Using the relation, $\widehat{\rho}_{i}(x)=\sum_{n} e^{i n \theta(x)}$, and performing the average, one arrives at the result (29). 


\section{REFERENCES}

[1] Mesoscopic Phenomena in Solids, B. L. Altshuler, P. A. Lee, and R. A. Webb, eds., (North Holland, 1991)

[2] R. Landauer, in: Localization, Interaction and Transport Phenomena, eds. B. Kramer, G. Bergmann and Y. Bruynsraede (Springer, Heidelberg, 1985) Vol.61, 38;

R. Landauer, in: W. van Haeringen and D. Lenstra (eds.), Analogies in Optics and Micro Electronics, 243-257, Kluwer Academic Publishers (1990);

Y. Imry, Directions in Condensed Matter Physics, 101, G. Grinstein and G. Mazenko, eds., (World Scientific, Singapore, 1986)

[3] G. B. Lesovik, JETP Letters 49, 594 (1989)

[4] B. Yurke and G. P. Kochanski, Phys. Rev. B41, 8184 (1990)

[5] M. Büttiker, Phys. Rev. Lett. 65, 2901 (1990); Phys. Rev. B46, 12485 (1992)

[6] S.-R. E. Yang, Solid State Commun.81, 375 (1992)

[7] C. W. J. Beenakker, M. Büttiker, Phys. Rev. B46, 1889 (1992)

[8] Th. Martin and R. Landauer, Phys. Rev. B45, 1742 (1992)

[9] M. J. M. de Jong and C. W. J. Beenakker, Phys. Rev. B46, 13400 (1992)

[10] L. S. Levitov and G. B. Lesovik, JETP Letters 58 (3), 230-235 (1993)

[11] L. S. Levitov and G. B. Lesovik, Quantum Measurement in Electric Circuit, preprint cond-mat/9401004

[12] D. A. Ivanov and L. S. Levitov, JETP Letters 58(6), 461 (1993) 
[13] H.-W. Lee and L. S. Levitov, Orthogonality catastrophe in a mesoscopic conductor due to a time-dependent flux, preprint cond-mat/9312013

[14] B. L. Altshuler, L. S. Levitov, and A. Yu. Yakovets, JETP Letters 59, 857 (1994) (in Russian: Pis'ma v ZhETF, vol. 59, 821 (1994))

[15] L. S. Levitov and G. B. Lesovik, Phys. Rev. Lett. 72, 538 (1994)

[16] D. A. Ivanov, H.-W. Lee, and L. S. Levitov, Coherent states of alternating current, preprint cond-mat/9501040, to appear in Phys. Rev. B

[17] H.-W. Lee and L. S. Levitov, Estimate of minimal noise in a quantum conductor, preprint cond-mat/9507011

[18] R. J. Glauber, Phys. Rev. Lett. 10, 84 (1963); Phys. Rev. 130, 2529 (1963);

L. Mandel and E. Wolf, Rev. Mod. Phys. 37, 231 (1965)

[19] C. W. Gardinger, Quantum Noise, Chapter 8, Springer-Verlag (1991);

J. R. Klauder and E. C. G. Sudarshan, Fundamentals of Quantum Optics, Chapter 8, W.A.Benjamin, Inc., N.Y. (1968)

[20] R. H. Koch, D. van Harlingen, and J. Clarke, Phys. Rev. B26, 74 (1982)

[21] S. Saito, et al., Phys. Lett. A162, 442 (1992)

[22] A. J. Leggett, Progr. Theor. Phys. Suppl.69, 80 (1980)

[23] M. Büttiker, Phys. Rev. B27, 6178 (1983);

A. I. Baz', Sov. Phys. JETP 20, 1261 (1965);

A. I. Baz', Ya. B. Zeldovich and A. M. Perelomov, Scattering, Reactions and De- 
cay in Nonrelativistic Quantum Mechanics, Israel Program for Scientific Translations, Jerusalem (1969)

[24] G. D. Mahan, Many Particle Physics, Secs. 4.4, 8.3, (2-nd edition, Plenum Press, 1990)

[25] K. D. Schotte and U. Schotte, Phys. Rev. 182, 479 (1969);

[26] F. D. M. Haldane, J. Phys. C14, 2585-2609 (1981)

[27] P. W. Anderson, Phys. Rev. Lett. 18, 1049 (1967)

[28] P. Nozières, C. T. deDominicis, Phys. Rev. 178, 1084 (1969)

[29] A. A. Belavin and A. M. Polyakov, Pis'ma ZhETF 22, 503 (1975);

see also: A. M. Polyakov, Gauge Fields and Strings, Chap.6, Sect.1 (Harwood Academic Publishers, 1987) 